\title{
DIABETES MELLITUS EN PEDIATRÍA
}

\author{
DIABETES MELLITUS IN PEDIATRICS
}

Los estudios de incidencia de diabetes mellitus tipo 1 (DM1) en Pediatría son de importancia para realizar un diagnóstico de la situación epidemiológica y posibilitar la elaboración de políticas sanitarias eficientes. La variación geográfica en incidencia refleja un grado diferente de susceptibilidad a la diabetes entre poblaciones. Aunque la susceptibilidad genética es necesaria para el desarrollo de la DM1, su etiología es multifactorial'.

Se ha descripto un aumento sostenido de la incidencia en poblaciones y en algunos grupos etarios, en ocasiones con características distintas en diferentes informes ${ }^{2}$. El análisis pormenorizado de los mismos podría generar interesantes hipótesis de trabajo en relación a factores ambientales que influirían en el desencadenamiento de la diabetes y favorecer su prevención. Varios de estos factores se analizan en trabajos de seguimiento como el BABYDIAB ${ }^{3}$ y elTEDDY, entre ellos, la alimentación, el nivel de vitaminas, el microbioma y el uso de vacunas ${ }^{4,5}$. Un registro en Alabama relaciona la introducción de la vacuna del rotavirus como una de las posibles causas de la disminución de la incidencia de DM1 en años recientes ${ }^{6}$.

Es interesante considerar los cambios de la incidencia en poblaciones con el paso de años. Recordemos que los registros son geográficos, de poblaciones que viven en un determinado espacio y tiempo. Hacia los años 1990 se consideraba que esto orientaba a una genética compartida, en particular en los países nórdicos. Si bien las migraciones nos acompañan desde el inicio de la historia, en los últimos años la globalización y las crisis sociales determinaron un cambio en la composición genética en grupos que comparten áreas geográficas. Esto puede influir en la incidencia y también en que grupos de menor riesgo genético aumenten la misma.

En un reciente artículo de la Federación Internacional de Diabetes (FID) se informa que en la región SACA (América del Sur y Central), 12 de 19 países cuentan con datos de incidencia?. Desgraciadamente en nuestro país son pocos los estudios recientes. Las dificultades que debemos superar para lograr registros actualizados son múltiples: el fraccionamiento del sistema de salud, las dificultades económicas y particularmente un orden de prioridades que no siempre favorece los registros prolijos y confiables.

El artículo publicado en esta edición -de las Dras. Gorbán de Lapertosa y Pomares, y los Dres. López y González- referido a la incidencia en niños menores de 14 años en la provincia de Corrientes, no sólo nos aporta datos concretos en cuanto a la evolución en el tiempo de la incidencia, sino que también intenta relacionar la misma con factores ambientales y personales. El prolijo trabajo realizado podría ser un punto de partida para la elaboración de registros nacionales absolutamente necesarios.

Dra. Mabel Ferraro Jefa del Servicio de Nutrición y Diabetes Hospital Gral. de Niños Pedro de Elizalde, Subdirectora de la Carrera de Médico Pediatra, Universidad de Buenos Aires (UBA) 


\section{BIBLIOGRAFÍA}

1. Karvonen M, Viik-Kajander M, Moltchanova E, Libman I, LaPorte R, Tuomilehto J. Incidence of childhood type 1 diabetes worldwide. Diabetes Mondiale (DiaMond) Project Group. Diabetes Care 2000 Oct; 23(10):1516-26.

2. Patterson CC, Dahlquist G, Gyuerues E, Soltész G. EURODIAB childhood type 1 diabetes registers. Results from the first 20 years. Conference paper in Diabetologia; Sep 2011.

3. Hummel S, Ziegler AG. Early determinants of type 1 diabetes: experience from the BABYDIAB and BABYDIET studies. Am J Clin Nutr 2011 Dec; 94(6 Suppl):1821S$1823 S$.

4. Mattila M, Erlund I, et al. Plasma ascorbic acid and the risk of islet autoimmunity and type 1diabetes: the TEDDY study. Diabetologia 2019. Disponible en: https:// doi.org/10.1007/s00125-019-05028-z
5. Lindfors K, Lin j, Lee HS, et al. Metagenomics of the faecal virome indicate a cumulative effect of enterovirus and gluten amount on the risk of coeliac disease autoimmunity in genetically at risk children: the TEDDY study. Gut 2019 Nov. Doi:10.1136/gutjnl-2019-319809

6. CorreyaTA, Ashraf AP, Griffin R, McCormick K, et al.Temporal trends in incidence of pediatric type 1 diabetes in Alabama: 2000-2017. Pediatric Diabetes 2019; 1-8. Doi: 10.1111/pedi.12927.

7. Patterson C, Dahlquist G, Soltesz G, et al. Worldwide estimates of incidence, prevalence and mortality of type 1 diabetes in children and adolescents. Results from the International Diabetes Federation Diabetes Atlas, $9^{\text {th }}$ Edition. Diabetes Research and Clinical Practice 2019; Vol 157. Doi: https://doi.org/10.1016/j.diabres.2019.107842. 